\title{
The QCD equation of state at finite density from analytical con- tinuation
}

\author{
Jana Günther ${ }^{1, a}$, Rene Bellwied ${ }^{2}$, Szabolcs Borsanyi ${ }^{1}$, Zoltan Fodor ${ }^{1,3,4}$, Sandor D. Katz ${ }^{3,5}$, Attila \\ Pasztor $^{1}$, and Claudia Ratti ${ }^{2}$ \\ ${ }^{1}$ Department of Physics, University of Wuppertal, Gaussstr. 20, D-42119 Wuppertal, Germany \\ ${ }^{2}$ Department of Physics, University of Houston, Houston, TX 77204, USA \\ ${ }^{3}$ Inst. for Theoretical Physics, Eötvös University \\ ${ }^{4}$ Jülich Supercomputing Centre, Forschungszentrum Jülich, D-52425 Jülich, Germany \\ ${ }^{5}$ MTA-ELTE "Lendület" Lattice Gauge Theory Research Group, Budapest, Hungary
}

\begin{abstract}
An efficient way to study the QCD phase diagram at small finite density is to extrapolate thermodynamical observables from imaginary chemical potential. In this talk we present results on several observables for the equation of state to order $\left(\mu_{B} / T\right)^{6}$. The observables are calculated along the isentropic trajectories in the $\left(T, \mu_{B}\right)$ plane corresponding to the RHIC Beam Energy Scan collision energies. The simulations are performed at the physical mass for the light and strange quarks. $\mu_{S}$ was tuned in a way to enforce strangeness neutrality to match the experimental conditions; the results are continuum extrapolated using lattices of up to $N_{t}=16$ temporal resolution.
\end{abstract}

\section{Introduction}

To analyse the quark gluon plasma that is created in heavy ion collision experiments at LHC or RHIC a theoretical understanding of the quark gluon plasma in QCD is needed. Around the deconfinement transition lattice simulations are the only systematic tool to study QCD since this region can not be accessed perturbatively. The investigation of the phase diagram at $\mu_{B} \neq 0$ is hindered by the sign problem. There have been several ideas in lattice QCD how to obtain results at real finite chemical potential like reweighting techniques [1-4], Taylor expansion [5-9], density of state methods [10, 11], using the canonical ensemble [12-14], the formulation with dual variables [15], Lefschetz thimbles $[16,17]$ or complex Langevin $[18,19]$. Unfortunately these techniques are not yet able to solve the sign problem completely, therefore at the moment results that are continuum extrapolated and obtained at physical quark masses are restricted to vanishing or imaginary chemical potential. Nevertheless one needs information also at $\mu_{B}>0$ particularly to analyse the collisions at RHIC [20]. In this work we use the method analytic continuation based on ensembles produced at imaginary chemical potential.

In terms of the baryon chemical potential $\mu_{B}$ the pressure $P$ can be written as a function of temperature $T$ :

$$
\frac{P\left(\mu_{B}, T\right)}{T^{4}}=c_{0}(T)+c_{2}(T)\left(\frac{\mu_{B}}{T}\right)^{2}+c_{4}(T)\left(\frac{\mu_{B}}{T}\right)^{4}+c_{6}(T)\left(\frac{\mu_{B}}{T}\right)^{6}+c_{8}(T)\left(\frac{\mu_{B}}{T}\right)^{8}+O\left(\mu_{B}^{10}\right)
$$

\footnotetext{
ae-mail: Jana.Guenther@t-online.de
} 
$c_{2}$ was calculated in the continuum in [21]. In [22] the coefficients $c_{2}$ to $c_{6}$ were presented on $N_{t}=6$ and $N_{t}=8$ lattices. In this proceedings we will go beyond those results and present the continuum extrapolated Taylor coefficients of the pressure up to $c_{6}$ based on [23]. Our analysis is done with

$$
\left\langle n_{S}\right\rangle=0 \text { and }\left\langle n_{Q}\right\rangle=0.4\left\langle n_{B}\right\rangle \text {. }
$$

\section{Simulation details}

We use a tree level Symanzik improved gauge action. The $2+1+1$ dynamical flavours are simulated with four times stout smeared staggered fermions [24]. The smearing parameter is $\rho=0.125$.

Our $T=0$ simulations can be described as follows. The up and the down quarks have degenerate masses $m_{u d}$. Both $m_{u d}$ and the strange quark mass $m_{s}$ are tuned in a way that the pion and the kaon mass divided by the pion decay constant are set to their physical value for every lattice spacing. The charm mass $m_{c}$ is set to be $m_{c} / m_{s}=11.85$ as described in [25]. For setting the scale in physical units we use $f_{\pi}$ or $w_{0}$ with lattice spacings between $a=0.063 \mathrm{fm}$ and $a=0.2 \mathrm{fm}$. For all of our lattices $L m_{\pi}>4$ is fulfilled.

For the finite temperature runs we use non vanishing imaginary chemical potentials. Due to the Roberge-Weiss transition $\mu_{B}$ has to be smaller then i $\pi T$ in these runs (see for example [26-28]). Our values for $\mu_{B}$ are chosen as

$$
\mu_{B}^{(j)}=\mathrm{i} T \frac{j}{8} \pi
$$

for $j \in\{0,3,4,5,6,7\}$. We tune $\mu_{S}$ to maintain strangeness neutrality. More details on this procedure can be found in $[29,30]$. For each value of $\mu_{B}$ we have simulations for sixteen temperatures between $135 \mathrm{MeV}$ and $210 \mathrm{MeV}$ on lattices with sizes $40^{3} \times 10,48^{3} \times 12$ and $64^{3} \times 16$. In addition, we produced configurations with $j=6.5$ on lattices with sizes $40^{3} \times 10$ and $48^{3} \times 12$. For each set of parameters we generated between 10000 and 20000 Hybrid Monte Carlo trajectories for the finite density configurations and 5 to 10 times more statistics for the $\mu_{B}=0$ runs. For $N_{t}=10$ and 12 every 5 th configuration is included in our study. For $N_{t}=16$ we analyse every 10th configuration. Though our simulations range up to a temperature of $210 \mathrm{MeV}$ we use these ensembles to determine the equation of state by analytic continuation. In order to extend the equation of state up to approximately $300 \mathrm{MeV}$ where the system is clearly in a state of quark gluon plasma, we collect data at these temperatures with $\mu_{B}=0^{1}$. To reach $300 \mathrm{MeV}$ we construct some extrapolated data. This means we are analysing a dataset

$$
\left.\frac{\mathrm{d} \hat{P}}{\mathrm{~d} \hat{\mu}_{B}}\right|_{\hat{\mu}_{B}}=\left\{\begin{array}{ll}
\left.\frac{\mathrm{d} \hat{P}}{\mathrm{~d} \hat{\mu}_{B}}\right|_{\text {simulated at } \hat{\mu}_{B}} & \text { for } T<T_{\text {conn }} \\
\left.\frac{\mathrm{d} \hat{P}}{\mathrm{~d} \hat{\mu}_{B}}\right|_{\text {extrapolated to } \hat{\mu}_{B}} & \text { for } T \geq T_{\text {conn }}
\end{array} .\right.
$$

Here the hat over a quantity means that it has been divided by the appropriate factors of $T$ to make it dimensionless. To check whether it is safe to neglect higher order contributions we will use two different connection points $T_{\text {conn }}$ from which on we will use the extrapolated data: One at $200 \mathrm{MeV}$ and one at $220 \mathrm{MeV}$. The difference in this two analysises will then add to our systematic error.

\footnotetext{
${ }^{1}$ In principle one may apply the Taylor expansion technique $[21,22]$ using these configurations. We did not follow this path.
} 


\section{Analysis}

As the simulations on the different lattice sizes correspond to slightly different temperatures and we want to know the derivative with respect to $T$, a first step in the analysis is the interpolation of $\frac{n}{\mu_{B} T^{2}}$, $\frac{n_{B}}{\mu_{B} T^{2}}$ and $\frac{\mathrm{d} \mu_{Q}}{\mathrm{~d} \mu_{B}}$ for every $\mu_{B}^{(j)}$. We use four different fit function:

$$
\begin{aligned}
& A_{1}(T)=a+b(T-f)+\frac{c}{T-f}+d \arctan (e(T-f)) \\
& A_{2}(T)=a+b(T-f)+\frac{c}{T-f}+\frac{d}{\left(1+|e(T-f)|^{g}\right)^{1 / g}}, \\
& A_{3}(T)=a+b(T-f)+c(T-f)^{2}+d \arctan (e(T-f)) \\
& A_{4}(T)=a+b(T-f)+c(T-f)^{2}+\frac{d}{\left(1+|e(T-f)|^{g}\right)^{1 / g}} .
\end{aligned}
$$

The fit functions are purely meant as interpolation and can not be extended beyond the fit range. From here on we will analyse the different temperatures separately.

The next step in the analysis is to fit the data in the $\hat{\mu}_{B}^{2}$ direction. For this we use three different fit functions:

$$
\begin{aligned}
& B_{1}(\hat{\mu})=a+b \hat{\mu}^{2}+c \hat{\mu}^{4}, \\
& B_{2}(\hat{\mu})=\frac{a+b \hat{\mu}^{2}}{1+c \hat{\mu}^{2}}, \\
& B_{3}(\hat{\mu})=a+b \hat{\mu}^{2}+c \frac{\sin (\hat{\mu})}{\hat{\mu}} .
\end{aligned}
$$

For imaginary $\mu_{B}$ all three fit functions describe the data well. However, their extrapolation to real $\mu_{B}$ can be different as shown in figure 1. These differences are included in our systematic uncertainties. Having obtained the $\mu_{B}>0$ data the next step is the continuum extrapolation. We use a linear fit as a function of $1 / N_{t}^{2}$ though the data from $N_{t}=10,12$ and 16. As an alternative analysis we also combine the the fit in the $\mu_{B}$ direction and the continuum extrapolation. In this case we use the three fit functions

$$
\begin{aligned}
& B_{1}(\hat{\mu})=a_{1}+\frac{a_{2}}{N_{t}^{2}}+\left(b_{1}+\frac{b_{2}}{N_{t}^{2}}\right) \hat{\mu}^{2}+\left(c_{1}+\frac{c_{2}}{N_{t}^{2}}\right) \hat{\mu}^{4}, \\
& B_{2}(\hat{\mu})=\frac{a_{1}+\frac{a_{2}}{N_{t}^{2}}+\left(b_{1}+\frac{b_{2}}{N_{t}^{2}}\right) \hat{\mu}^{2}}{1+\left(c_{1}+\frac{c_{2}}{N_{t}^{2}}\right) \hat{\mu}^{2}}, \\
& B_{3}(\hat{\mu})=a_{1}+\frac{a_{2}}{N_{t}^{2}}+\left(b_{1}+\frac{b_{2}}{N_{t}^{2}}\right) \hat{\mu}^{2}+\left(c_{1}+\frac{c_{2}}{N_{t}^{2}}\right) \frac{\sin (\hat{\mu})}{\hat{\mu}} .
\end{aligned}
$$

From equation (9) to (11) or from equation (12) to (14) we can determine the Taylor coefficients of the pressure. The results are shown in figure 2. The statistical error is determined via the bootstrap method with 1000 bootstrap samples. 


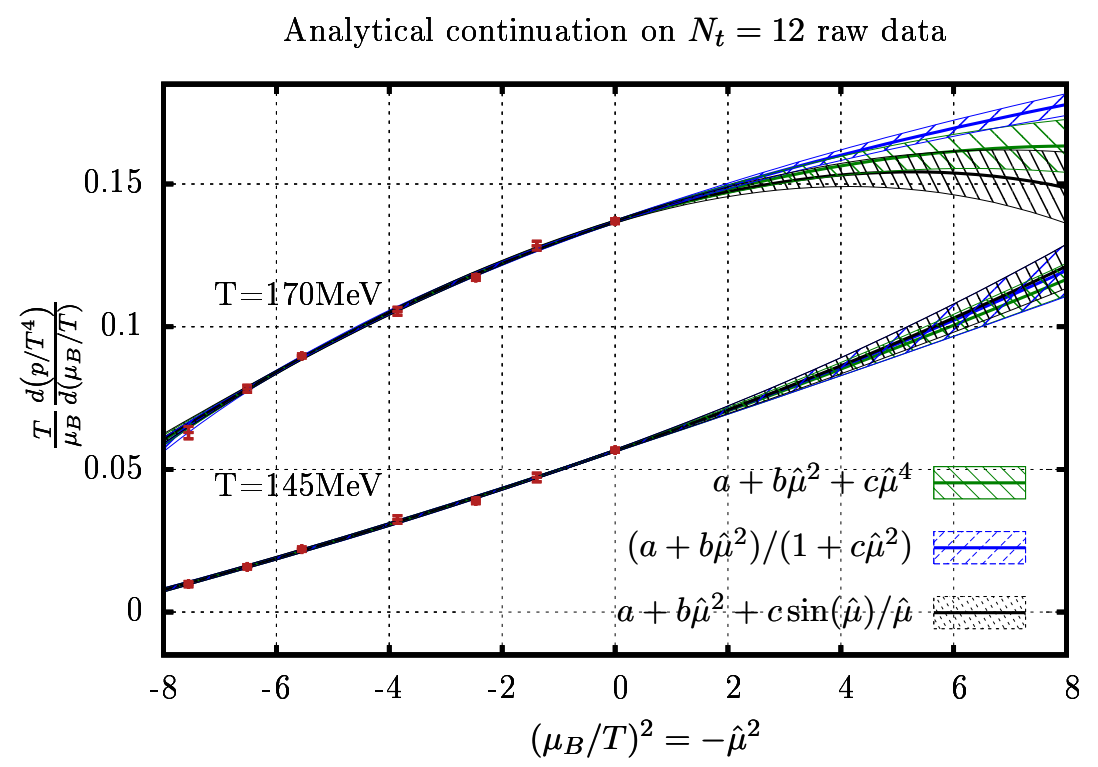

Figure 1. The increasing error at larger $\mu_{B}$ from the three different fit functions depending on the temperature. While at $\mu_{B}<0$ the fit functions look very similar they can still differ at positive $\mu_{B}$. Here the data from the $N_{t}=12$ lattice is shown as this allows a comparison to the raw data.

For the systematic error we use the histogram method of $[31,32]$. In this case we have the following options to chose from for different analysises:

- two methods of scale setting

- four fits in the $T$ direction

- three fits in the $\mu_{B}$ direction

- combined and separate $\mu_{B}$-fit and continuum extrapolation

- two different points $T_{\text {conn }}$ from which we use the data extrapolated from $\mu_{B}=0$ at high temperatures.

This adds up to a total of $2 \cdot 4 \cdot 3 \cdot 2 \cdot 2=96$ analysises. The systematic uncertainty is defined by the central $68 \%$ of the distribution. Figure 2 shows the result with statistical and the systematic error added quadratically.

The importance of the higher order terms can be seen from figure 3. From the Taylor coefficient we can calculate $\hat{n}_{B}$ to different orders. Though $c_{2}$ gives the pressure up to $O\left(\mu_{B}^{2}\right)$ it proviedes $\hat{n}_{B}$ only up to $O\left(\mu_{B}\right)$. The reason for that is that $\hat{n}_{B}$ is obtained as a derivative with respect to $\mu_{B}$. For a temperature of $145 \mathrm{MeV}$ only the $c_{2}$ and $c_{4}$ coefficient contribute to the result because $c_{6}$ is tiny. At this temperature the analytic continuation for $\hat{n}_{B}$ up to order $O\left(\mu_{B}^{5}\right)$ only increases the error compared to the previous order. This is not surprising as figure 2 shows that at $T=145 \mathrm{MeV} c_{6}$ is compatible with zero. However at a temperature where $c_{6}$ differs significantly from zero, for example $170 \mathrm{MeV}$, the additional order has a visible influence.

Besides the Taylor coefficients $c_{2} \ldots c_{6}$ we compute the equation of state. In heavy ion collisions if one neglects dissipative effects, the quark gluon plasma created in the collision expands with a fixed 

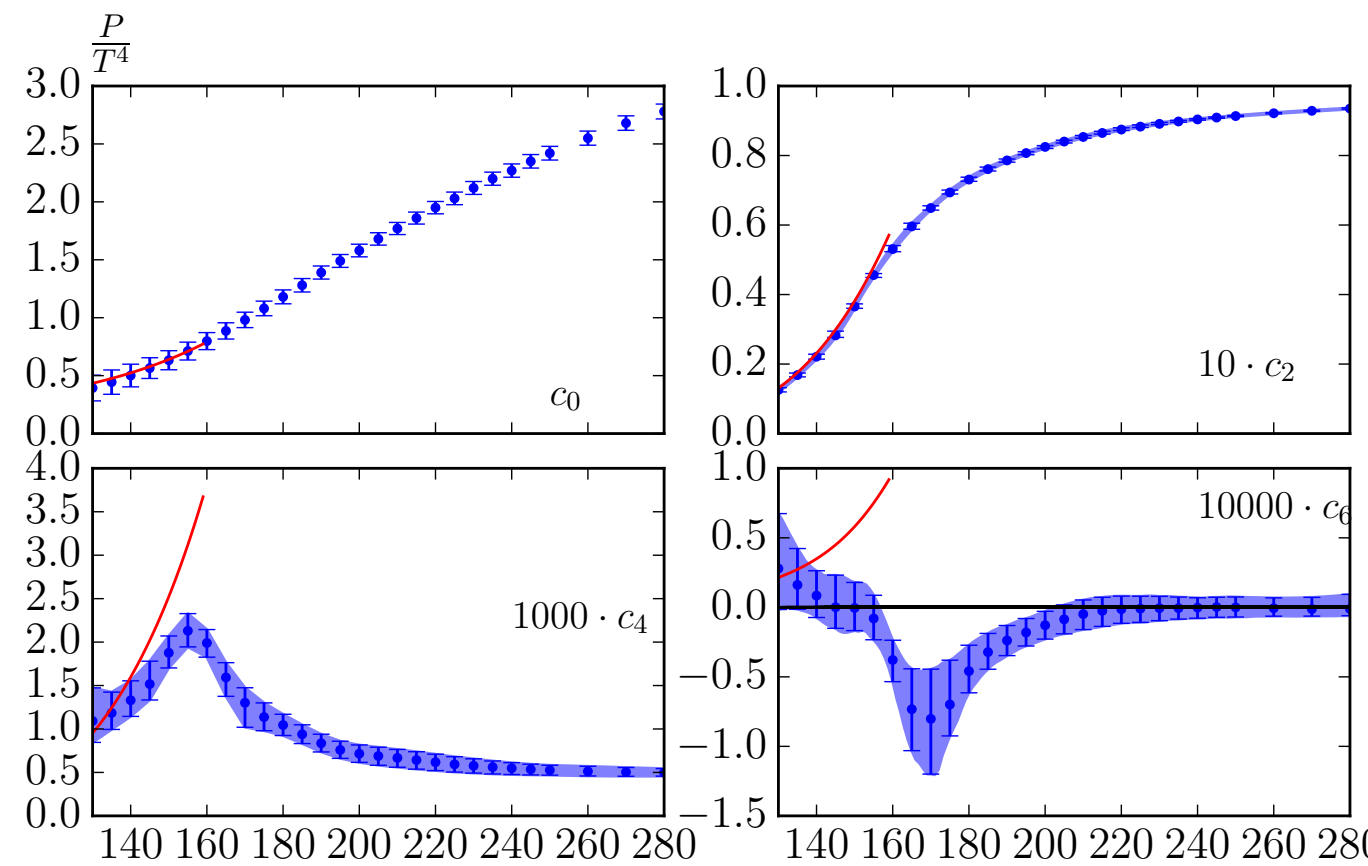

$$
\frac{T}{\mathrm{MeV}}
$$
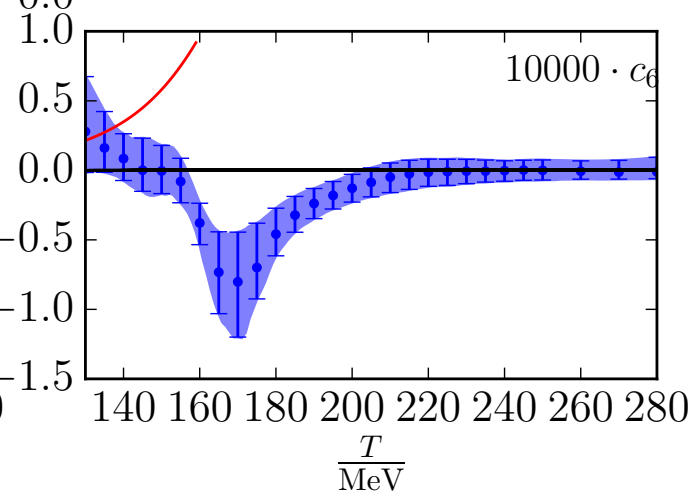

Figure 2. The Taylor coefficients of the pressure. The values for $c_{0}$ are taken from [33]. The red line shows the expectations of the HRG model.

baryon number and without generation of entropy. Therefore the ratio $\frac{S}{N_{B}}$ is constant. The medium cools down along these isentropic trajectories in the $T$ - $\mu_{B}$-plane. Therefore we compute the equation of state along these trajectories. In figure 4 we show six of them which were matched to the beem energies of RHIC as determined in [34]. The solid lines are predictions of the HRG model that we use to continue our trajectories at lower temperatures. The $\frac{S}{N_{B}}=30$ choice corresponds to a beam energy of $14.5 \mathrm{GeV}$. As it can be seen from the errors this is at the end of how far we can extrapolate in $\mu_{B}$. Since the error for this case is relatively large we compute the equation of state along the trajectories with $\frac{S}{N_{B}}=51$ and $\frac{S}{N_{B}}=420$. Figure 5 shows the pressure and the trace anomaly along these isentropic trajectories.

\section{Conclusion}

We investigated the equation of state around the QCD transition temperature at finite baryon chemical potential with lattice QCD. Since this can not be done directly due to the sign problem, we used simulations at imaginary baryon chemical potential to analytically continue to real chemical potentials. We determined the Taylor coefficients of the pressure up to $O\left(\mu_{B}^{6}\right)$ in the continuum limit. Results up to $\mu_{B} / T \approx 2$ were given. All simulations were done at the strangeness neutral point to match conditions in heavy ion collision experiments. We then calculated the pressure and the trace anomaly along the isentropic trajectories. The trajectories were matched to the RHIC beam energies. 

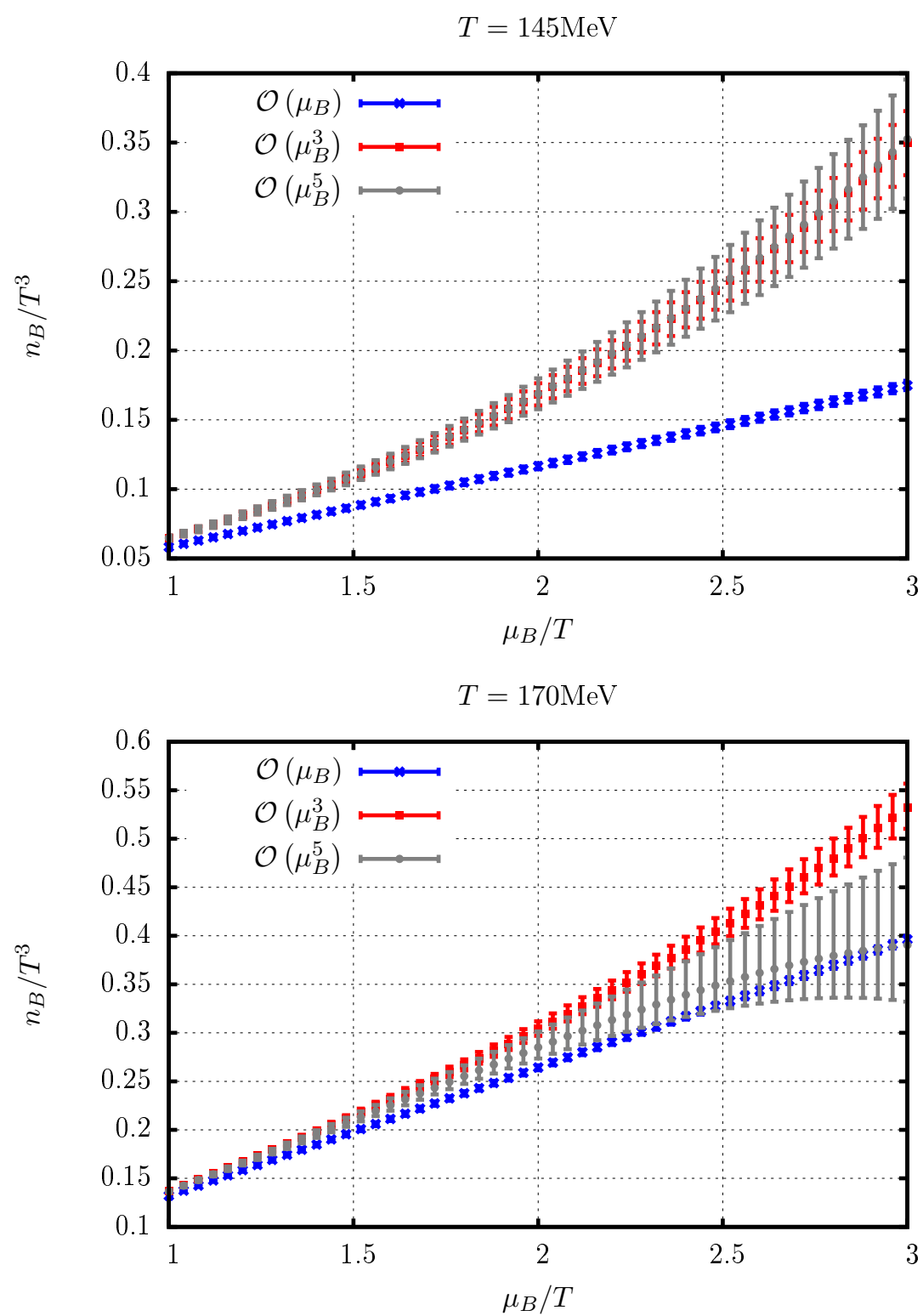

Figure 3. The contribution of the different order coefficients to the baryon number. At $145 \mathrm{MeV}$ the contribution of $c_{6}$ is negligible while at $170 \mathrm{MeV}$ it influences the results. 


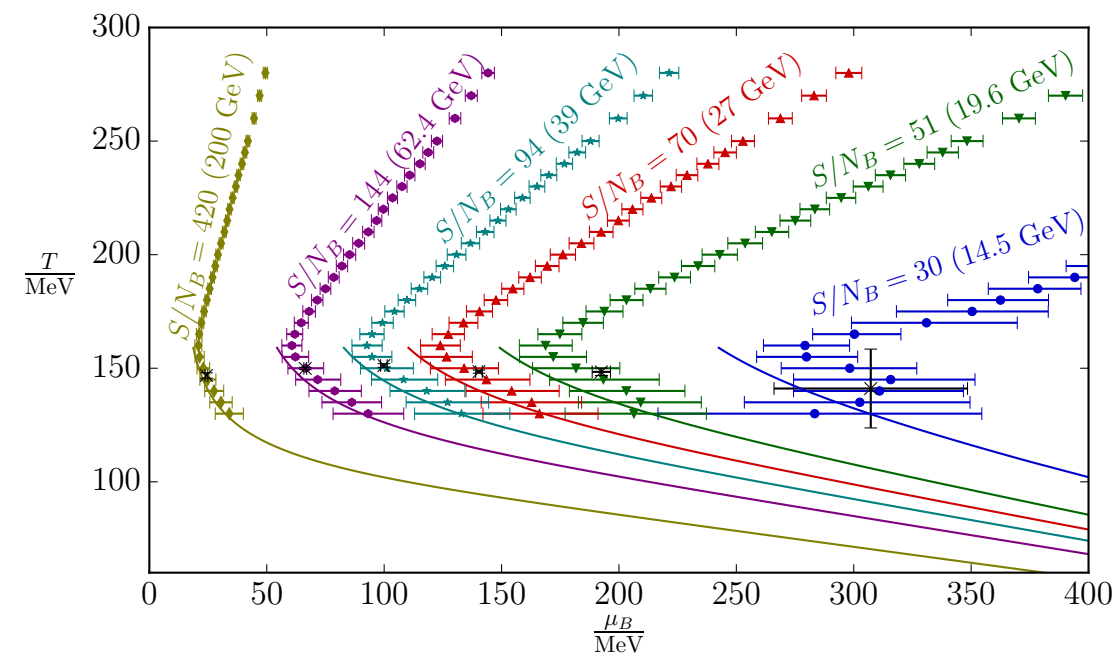

Figure 4. The trajectories of constant $\frac{S}{N_{B}}$ matched to the beam energies of RHIC as determined in [34].

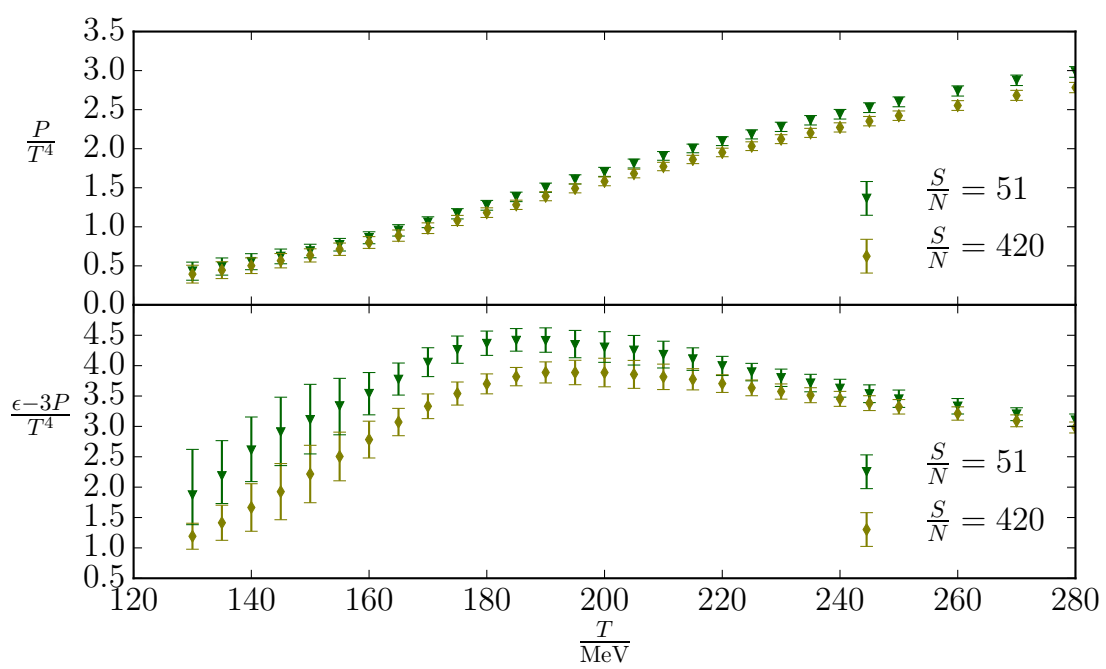

Figure 5. The pressure and the trance anomaly along trajectories of constant $\frac{S}{N}$. 


\section{Acknowledgements}

C.R. would like to thank Volker Koch, Jacquelyn Noronha-Hostler, Jorge Noronha and Bjorn Schenke for fruitful discussions. This project was funded by the DFG grant SFB/TR55. This material is based upon work supported by the National Science Foundation through grant number NSF PHY-1513864 and by the U.S. Department of Energy, Office of Science, Office of Nuclear Physics, within the framework of the Beam Energy Scan Theory (BEST) Topical Collaboration. An award of computer time was provided by the INCITE program. This research used resources of the Argonne Leadership Computing Facility, which is a DOE Office of Science User Facility supported under Contract DEAC02-06CH11357. The authors gratefully acknowledge the Gauss Centre for Supercomputing (GCS) for providing computing time for a GCS Large-Scale Project on the GCS share of the supercomputer JUQUEEN [35] at Jülich Supercomputing Centre (JSC).

\section{References}

[1] I.M. Barbour, S.E. Morrison, E.G. Klepfish, J.B. Kogut, M.P. Lombardo, Nucl. Phys. Proc. Suppl. 60A, 220 (1998), hep-lat/9705042

[2] Z. Fodor, S.D. Katz, Phys. Lett. B534, 87 (2002), hep-lat/0104001

[3] Z. Fodor, S.D. Katz, JHEP 03, 014 (2002), hep-lat/0106002

[4] F. Csikor, G.I. Egri, Z. Fodor, S.D. Katz, K.K. Szabo, A.I. Toth, Nucl. Phys. Proc. Suppl. 119, 547 (2003), [,547(2002)], hep-lat/0209114

[5] C.R. Allton, S. Ejiri, S.J. Hands, O. Kaczmarek, F. Karsch, E. Laermann, C. Schmidt, L. Scorzato, Phys. Rev. D66, 074507 (2002), hep-lat/0204010

[6] C.R. Allton, M. Doring, S. Ejiri, S.J. Hands, O. Kaczmarek, F. Karsch, E. Laermann, K. Redlich, Phys. Rev. D71, 054508 (2005), hep-lat/0501030

[7] R.V. Gavai, S. Gupta, Phys. Rev. D78, 114503 (2008), 0806. 2233

[8] S. Basak et al. (MILC), PoS LATTICE2008, 171 (2008), 0910.0276

[9] O. Kaczmarek, F. Karsch, E. Laermann, C. Miao, S. Mukherjee, P. Petreczky, C. Schmidt, W. Soeldner, W. Unger, Phys. Rev. D83, 014504 (2011), 1011.3130

[10] Z. Fodor, S.D. Katz, C. Schmidt, JHEP 03, 121 (2007), hep-lat/0701022

[11] A. Alexandru, C. Gattringer, H.P. Schadler, K. Splittorff, J.J.M. Verbaarschot, Phys. Rev. D91, 074501 (2015), 1411.4143

[12] A. Alexandru, M. Faber, I. Horvath, K.F. Liu, Phys. Rev. D72, 114513 (2005), hep-lat/0507020

[13] S. Kratochvila, P. de Forcrand, PoS LAT2005, 167 (2006), hep-lat/0509143

[14] S. Ejiri, Phys. Rev. D78, 074507 (2008), 0804 . 3227

[15] C. Gattringer, PoS LATTICE2013, 002 (2014), 1401.7788

[16] L. Scorzato, PoS LATTICE2015, 016 (2016), 1512.08039

[17] A. Alexandru, G. Basar, P. Bedaque, Phys. Rev. D93, 014504 (2016), 1510.03258

[18] E. Seiler, D. Sexty, I.O. Stamatescu, Phys. Lett. B723, 213 (2013), 1211. 3709

[19] D. Sexty, Phys. Lett. B729, 108 (2014), 1307.7748

[20] G. Odyniec, EPJ Web Conf. 95, 03027 (2015)

[21] S. Borsanyi, G. Endrodi, Z. Fodor, S.D. Katz, S. Krieg, C. Ratti, K.K. Szabo, JHEP 08, 053 (2012), 1204.6710

[22] P. Hegde (BNL-Bielefeld-CCNU), Nucl. Phys. A931, 851 (2014), 1408.6305 
[23] J. Gunther, R. Bellwied, S. Borsanyi, Z. Fodor, S.D. Katz, A. Pasztor, C. Ratti (2016), 1607.02493

[24] C. Morningstar, M.J. Peardon, Phys. Rev. D69, 054501 (2004), hep-lat/0311018

[25] C. McNeile, C.T.H. Davies, E. Follana, K. Hornbostel, G.P. Lepage, Phys. Rev. D82, 034512 (2010), 1004.4285

[26] M. D’Elia, F. Sanfilippo, Phys. Rev. D80, 111501 (2009), 0909.0254

[27] C. Pinke, O. Philipsen, PoS LATTICE2013, 209 (2014)

[28] L.K. Wu, X.F. Meng, Phys. Rev. D90, 094506 (2014), 1405. 2425

[29] R. Bellwied, S. Borsanyi, Z. Fodor, J. Günther, S.D. Katz, C. Ratti, K.K. Szabo, Phys. Lett. B751, 559 (2015), 1507.07510

[30] J. Günther, R. Bellwied, S. Borsanyi, Z. Fodor, S.D. Katz, C. Ratti, K. Szabo, PoS LATTICE2015, 142 (2016)

[31] S. Durr et al., Science 322, 1224 (2008), 0906.3599

[32] S. Borsanyi et al., Science 347, 1452 (2015), 1406. 4088

[33] S. Borsanyi, Z. Fodor, C. Hoelbling, S.D. Katz, S. Krieg, K.K. Szabo, Phys. Lett. B730, 99 (2014), 1309. 5258

[34] P. Alba, W. Alberico, R. Bellwied, M. Bluhm, V. Mantovani Sarti, M. Nahrgang, C. Ratti, Phys. Lett. B738, 305 (2014), 1403. 4903

[35] Jülich Supercomputing Centre, Journal of large-scale research facilities A1, 1 (2015) 